\title{
Diverging time scale in the dimensional crossover for liquids in strong confinement
}

\author{
Suvendu Mandal and Thomas Franosch \\ Institut für Theoretische Physik, Universität Innsbruck, Technikerstr. 21A, A-6020 Innsbruck, Austria
}

(Dated: November 12, 2018)

\begin{abstract}
We study a strongly interacting dense hard-sphere system confined between two parallel plates by event-driven molecular dynamics simulations to address the fundamental question of the nature of the $3 \mathrm{D}$ to $2 \mathrm{D}$ crossover. As the fluid becomes more and more confined the dynamics of the transverse and lateral degrees of freedom decouple, which is accompanied by a diverging time scale separating $2 \mathrm{D}$ from $3 \mathrm{D}$ behavior. Relying on the time-correlation function of the transversal kinetic energy the scaling behavior and its density-dependence is explored. Surprisingly, our simulations reveal that its time-dependence becomes purely exponential such that memory effects can be ignored. We rationalize our findings quantitatively in terms of an analytic theory which becomes exact in the limit of strong confinement.
\end{abstract}

Introduction. - Transport of particles in nanoconfinement is of great scientific and industrial importance with applications in heterogeneous catalysis [1], oil recovery [2], or lubrication [3-6]. In recent years artificial nanoporous materials such as metal organic frameworks $[7,8]$, zeolites $[9,10]$, and biocompatible scaffolds [11] have also triggered many novel applications, including gas storage [12], repairing or regenerating tissues [11], size-selective molecular sieving [13], lab-on-a chip technology and nanofluidics $[14,15]$. The efficiency of such nanodevices often crucially depends on higher surface to volume ratio, such that the distance between the confining walls may even reach atomic scale [16], or the system effectively becomes quasi-2D. Nevertheless, despite its long history, a deep understanding of the transport mechanisms in nanoconfinement and how the dimensional crossover occurs dynamically is still far from satisfactory.

Early theoretical studies on transport in nanoconfinement starting from Knudsen and Smoluchowski $[17,18]$ focused on dilute hard-sphere gases where exact results could be obtained analytically in the low-density limit [17-22] by assuming particle-wall collisions as diffusive. In contrast, confinement effects on dense strongly interacting systems have only recently come into focus [23]. There, the simplest geometry to investigate the effects of strong confinement is a slit where fluid particles are restricted to a narrow space between two smooth parallel plates, but also tubes or spherical confinements have been realized experimentally [24-26]. Computer simulations and experiments for the planar confinement have revealed an exotic equilibrium phase behavior due to commensurable stacking [27-34] as well as the hexatic phases in the limit of quasi-2D confinement $[35,36]$. Confinement induced order-disorder phase transitions for certain nonpolar liquids have also been reported in several experiments [37], but the interpretation has been challenged in favor of a glass transition [38-40]. The structural properties of strongly confined liquids have been measured directly only recently by x-ray scattering [41, 42].

The structural changes by the confinement also have

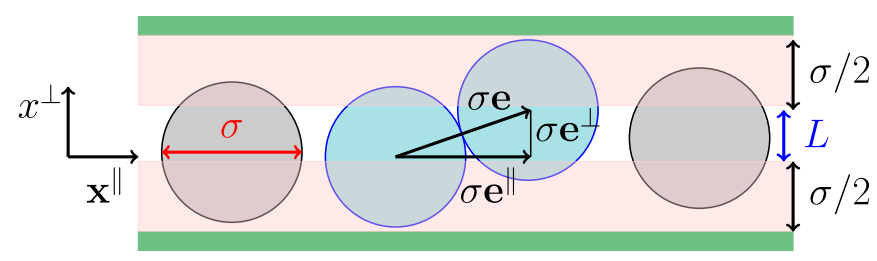

FIG. 1. Schematic cross section of hard spheres of diameter $\sigma$ confined to a slit of accessible width $L$. For two colliding spheres the velocity transfer is directed along the connecting vector $\sigma \mathbf{e}$ which lies almost parallel to the walls for small widths $L$.

drastic ramifications for the dynamic properties. For instance, the role of local order has been elucidated within a remarkable empirical scaling of the diffusivity or structural relaxation times with the excess entropy [43-46]. Complementarily, a microscopic theory for the dynamics in confinement is the mode-coupling theory that predicts a multiple-reentrant in a glassy phase as the plate separation is varied [33, 47, 48]

From a more fundamental point of view one would like to know how the dimensional crossover from a 3D bulk liquid to a (quasi-)2D system occurs. For the thermodynamic and structural properties it has been shown recently that a small parameter emerges such that the convergence to a $2 \mathrm{D}$ system including leading corrections can be proven [49]. The key observation there was that in strong confinement the canonical ensemble for the fluid in a slit geometry decouples into a two-dimensional fluid in the lateral plane and an ideal gas in the transversal direction. However, the consequences of the weak coupling between lateral and transversal degrees of freedom for slow equilibration and how time-dependent correlation functions will be affected by coupling to the 'other dimension' have remained mostly unexplored.

In this Letter, we now address the dynamical confinement problem and demonstrate that as the fluid becomes more and more confined a singular time scale emerges separating 2D from 3D behavior. The dependence of this divergent time scale on the plate separation and the pack- 
ing fraction will be worked out analytically including the prefactor and validated by simulations.

Simulation We investigate a fluid of hard spheres of diameter $\sigma$ confined between two flat parallel hard walls with accessible slit width $L$, see Fig. 1 . Trajectories are computed by event-driven molecular dynamics with initial velocities drawn from a Maxwell Boltzmann distribution with thermal velocity $v_{t h}=\sqrt{k_{B} T / m}$, which also sets the natural time scale $t_{0}=\sigma / v_{t h}$. We focus on small wall separations $L / \sigma=0.01, \ldots, 0.5<1$ such that only a single monolayer fits between the plates and consider systems at 2D packing fractions $\varphi_{2 D}=(\pi / 4) \sigma^{2} N / A=$ $0.1, \ldots, 0.8$ for $N$ particles per area $A$, such that the highest densities are already beyond the freezing transition.

To unravel the divergent time scale we choose an observable that displays nontrivial dynamics exclusively due to the weak coupling of the transversal to the lateral degrees of freedom. Since in the decoupled ensemble the lateral degrees of freedom evolve just like in a confined ideal gas, a natural candidate is the transversal kinetic energy $\epsilon_{s}^{\perp}(t)=(m / 2)\left[v_{s}^{\perp}(t)\right]^{2}$ of a tagged particle, i.e. any $s \in\{1, \ldots, N\}$, and $v_{s}^{\perp}(t)$ is the fluctuating velocity perpendicular to the plates. We therefore monitor the time-correlation function

$$
T_{s}^{\perp}(t)=\left\langle\delta \epsilon_{s}^{\perp}(t) \delta \epsilon_{s}^{\perp}(0)\right\rangle,
$$

of the fluctuations $\delta \epsilon_{s}^{\perp}(t)=\epsilon_{s}^{\perp}(t)-k_{B} T / 2$. From the Maxwell-Boltzmann distribution one readily computes its initial value $T_{s}^{\perp}(0)=\left\langle\left|\delta \epsilon_{s}^{\perp}\right|^{2}\right\rangle=\left(k_{B} T\right)^{2} / 2$.

The simulation results are displayed in Fig. 2(a) for the moderate packing fraction $\varphi_{2 D}=0.4$ (well below the two-dimensional freezing transition to a triangular phase $\left.\varphi_{2 \mathrm{D}}^{\text {freezing }} \approx 0.69[27,29,49]\right)$ for accessible plate separations covering two decades. One infers that the characteristic time scale increases by 4 orders of magnitude as the plate separation is decreased by a factor of 100 , while the shape of the relaxation function becomes independent of the plate distance for small $L$. This suggests that data collapse can be achieved upon proper rescaling with the measured relaxation time. Plotting the data on a semi$\log$ plot then demonstrates that for small $L$ the data follow a pure exponential $\exp (-t / \tau)$ even at the smallest time scales, see Fig. 2(b). Deviations become apparent only at the largest distance considered, $L / \sigma=1$.

The relaxation times $\tau=\tau\left(L, \varphi_{2 D}\right)$ extracted from the simulations, see Fig. 3, approach a divergence $\sim L^{-2}$ for all packing fractions considered, the power law being an excellent description of the data already at wall separations $L / \sigma \lesssim 0.5$. Dimensional analysis suggests that in this regime the relaxation rate should read

$$
\tau^{-1}=t_{0}^{-1}\left(\frac{L}{\sigma}\right)^{2} A\left(\varphi_{2 D}\right),
$$

where the prefactor $A\left(\varphi_{2 D}\right)$ depends on the packing fraction only. The prefactors $A\left(\varphi_{2 D}\right)$ as measured from the
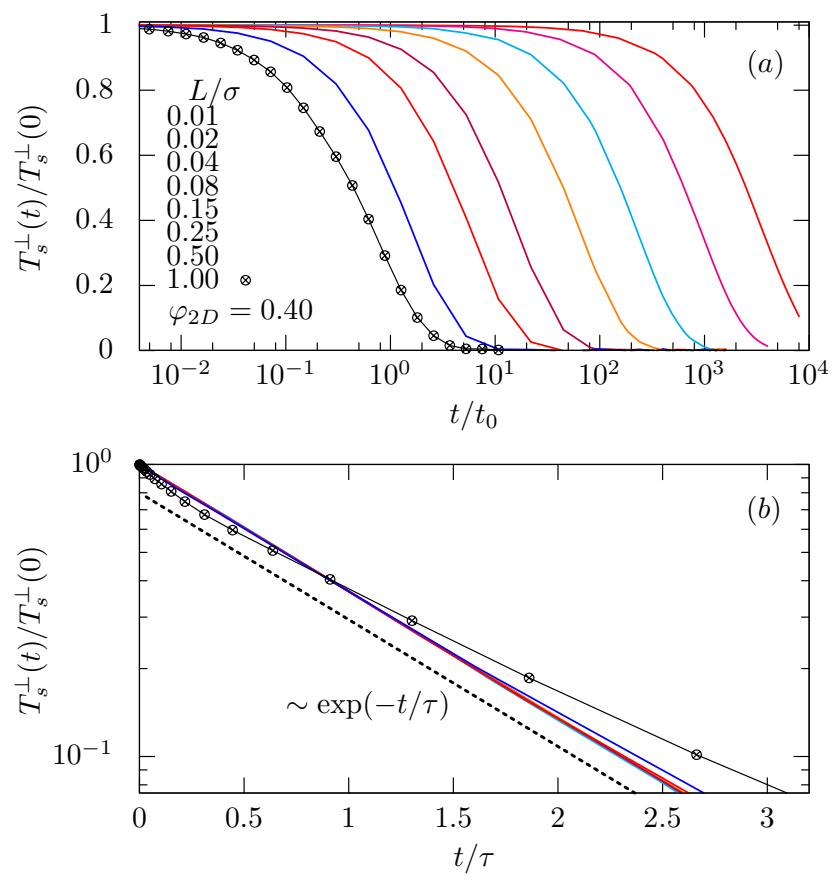

FIG. 2. (a) Decay of the time-correlation function $T_{s}^{\perp}(t)$ of the transversal kinetic energy for $\varphi_{2 D}=0.40$ and various wall separations $L$. Wall distance decreases from left to right. (b) Same data in semi-log plot after rescaling with the measured relaxation time $\tau$. As a guide to the eye, the dashed line indicates a pure exponential decay $\sim \exp (-t / \tau)$.

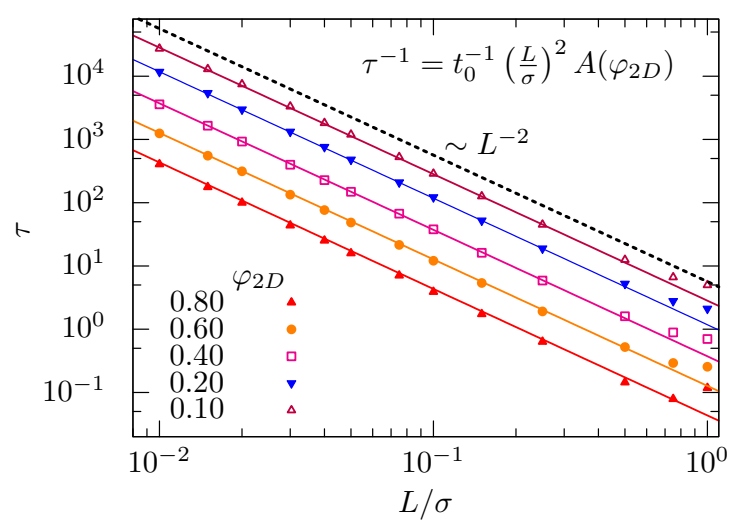

FIG. 3. Relaxation time $\tau$ for the strongly confined liquids as function of the wall separation $L$ for various packing fractions $\varphi_{2 D}$. Packing fraction increases from top to bottom. The dashed line is a power law $\sim L^{-2}$ and serves as a guide to the eye.

simulation data, displayed in Fig. 4, increases smoothly with the packing fraction, in particular, it grows stronger than the packing fraction itself.

Theory The coordinates and velocities of the particles split naturally into lateral and transversal degrees of freedom, $\mathbf{x}_{n}=\left(\mathbf{x}_{n}^{\|}, x_{n}^{\perp}\right), \mathbf{v}_{n}=\left(\mathbf{v}_{n}^{\|}, v_{n}^{\perp}\right), n=$ 


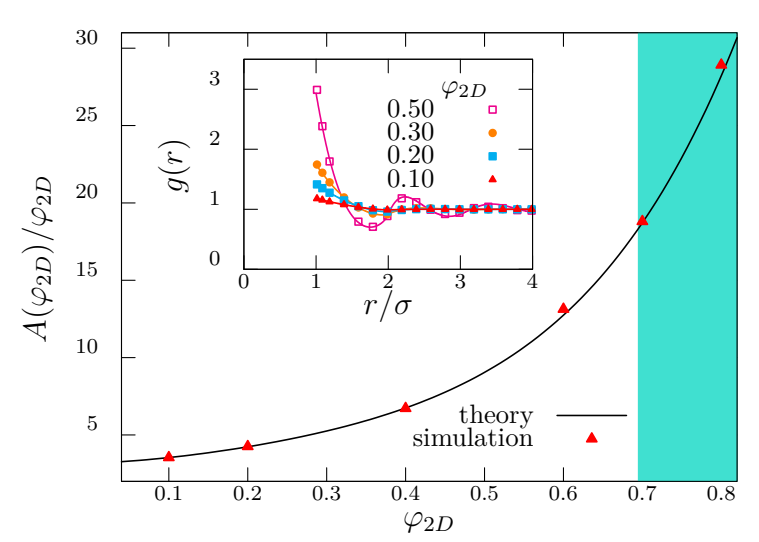

FIG. 4. Prefactor $A\left(\varphi_{2 D}\right)$ for the scaling behavior of the relaxation rate $\tau\left(L, \varphi_{2 D}\right)^{-1}=t_{0}^{-1}(L / \sigma)^{2} A\left(\varphi_{2 D}\right)$ as a function of $2 D$ packing fraction $\varphi_{2 D}$. The shaded area indicates the ordered phase. The inset shows the $2 D$ radial distribution function at some representative packing fractions. The solid lines are from the Ornstein-Zernike relation using the PercusYevick closure, whereas the symbols are from simulations.

$1, \ldots, N$, the confinement restricts the transversal coordinates to $\left|x_{n}^{\perp}\right| \leq L / 2$, see Fig. 1 . The time evolution of an observable $A(t)=A\left(\left\{\mathbf{x}_{n}(t)\right\},\left\{\mathbf{v}_{n}(t)\right\}\right)$ is inherited from the trajectories and is formally encoded in $A(t)=\exp \left(i \mathcal{L}_{ \pm} t\right) A, t \gtrless 0$, with the pseudo-Liouville operator $[50,51]$

$$
i \mathcal{L}_{ \pm}=\sum_{n} \mathbf{v}_{n} \cdot \frac{\partial}{\partial \mathbf{x}_{n}}+\sum_{n} \hat{W}_{ \pm}(n)+\sum_{m<n} \hat{T}_{ \pm}(m, n) .
$$

Here the first term describes merely the free-streaming motion, while the operator $\hat{W}_{ \pm}(n)$ in the second term accounts for the collision of particle $n$ with the hard walls. Explicit expressions are readily derived following the standard method [51] but won't be needed in the following. The interaction between the hard spheres is encoded in the binary collision operator

$$
\begin{aligned}
\hat{T}_{ \pm}(m n) & =\sigma^{2} \int \mathrm{d} \mathbf{e} \Theta\left(\mp \mathbf{v}_{m n} \cdot \mathbf{e}\right)\left|\mathbf{v}_{m n} \cdot \mathbf{e}\right| \\
& \times \delta^{(3)}\left(\mathbf{x}_{m n}-\sigma \mathbf{e}\right)\left[\hat{b}^{\mathbf{e}}(m n)-1\right] .
\end{aligned}
$$

Here $\mathbf{e}$ is a unit vector and the integral extends over the unit sphere. The normal component of the relative velocity of the colliding pair $\mathbf{v}_{m n} \cdot \mathbf{e}=\left(\mathbf{v}_{m}-\mathbf{v}_{n}\right) \cdot \mathbf{e}$ (multiplied by the infinitesimal time $\mathrm{d} t$ ) defines the collision cylinder. The Heaviside step function $\Theta(\cdot)$ selects approaching/distancing particles and the $\delta$-function ensures the contact condition for the collision and determines the unit vector $\mathbf{e}$. The operator $\hat{b}^{\mathbf{e}}(m n)$ acts only on the velocities $\mathbf{v}_{m}$ and $\mathbf{v}_{n}$ and replaces them by the velocities $\tilde{\mathbf{v}}_{m}, \tilde{\mathbf{v}}_{n}$ after the collision

$$
\begin{aligned}
b^{\mathbf{e}}(m n) \mathbf{v}_{m} & :=\tilde{\mathbf{v}}_{m}=\mathbf{v}_{m}-\mathbf{e}\left(\mathbf{e} \cdot \mathbf{v}_{m n}\right), \\
b^{\mathbf{e}}(m n) \mathbf{v}_{n} & :=\tilde{\mathbf{v}}_{n}=\mathbf{v}_{n}+\mathbf{e}\left(\mathbf{e} \cdot \mathbf{v}_{m n}\right)
\end{aligned}
$$

The coupling between the transversal and lateral degrees of freedom occurs only via the collisions $\hat{T}_{ \pm}(m n)$. Yet, if the accessible slit width is much smaller than the hard-sphere diameter, $L \ll \sigma$, the unit vector $\mathbf{e}=$ $\left(\mathbf{e}^{\|}, e^{\perp}\right)$ for the contact condition satisfies $\left|e^{\perp}\right| \leq L / \sigma \ll$ $1,\left|\mathbf{e}^{\|}\right| \simeq 1$, see Fig. 1, such that the momentum transfer is almost planar. This insight suggests that the collision operator may be replaced to leading order by its twodimensional analogue $\hat{T}_{ \pm}^{\|}(m n)$ where the contact condition $\delta^{(3)}\left(\mathbf{x}_{m n}-\sigma \mathbf{e}\right) \mapsto \delta^{(2)}\left(\mathbf{x}_{m n}^{\|}-\sigma \mathbf{e}^{\|}\right) \delta\left(\sigma e^{\perp}\right)$ in Eq. (4) is replaced by a collision within the plane. Then the pseudo-Liouville operator naturally decomposes

$$
i \mathcal{L}_{ \pm}=i \mathcal{L}_{ \pm}^{\perp}+i \mathcal{L}_{ \pm}^{\|}+i \mathcal{L}_{ \pm}^{\mathrm{int}},
$$

where $\mathcal{L}_{ \pm}^{\perp}$ accounts for the transversal degrees of freedom of a confined ideal gas, $\mathcal{L}_{ \pm}^{\|}$corresponds to the time evolution of a two-dimensional hard-disk fluid, while the residual interaction is encoded in

$$
i \mathcal{L}_{ \pm}^{\text {int }}=\sum_{m<n}\left[\hat{T}_{ \pm}(m, n)-\hat{T}_{ \pm}^{\|}(m, n)\right] .
$$

The idea is that $i \mathcal{L}_{ \pm}^{\text {int }}$ induces only a weak coupling. Ignoring this contribution leads to a decoupled ensemble of interacting lateral degrees of freedom and an ideal gas of transversal degrees of freedom. This weak coupling therefore introduces a time scale up to which the coupling of the degrees of freedom is irrelevant. As the plate separation becomes smaller this time scale is expected to grow.

The transversal kinetic energy is conserved in the decoupled ensemble $\partial_{t} \delta \epsilon_{s}^{\perp}=i \mathcal{L}_{ \pm}^{\text {int }} \delta \epsilon_{s}^{\perp}$ and the decay of its time correlation function $T_{s}^{\perp}(t)$ directly reflects the small coupling. Relying on the Zwanzig-Mori projection operator formalism $[52,53]$ an exact equation of motion (e.o.m) can be readily derived [54] for $t>0$

$$
\dot{T}_{s}^{\perp}(t)+\tau^{-1} T_{s}^{\perp}(t)+\int_{0}^{t} K_{s}^{\perp}\left(t-t^{\prime}\right) T_{s}^{\perp}\left(t^{\prime}\right) \mathrm{d} t^{\prime}=0 .
$$

Here, the second term describes an instantaneous relaxation, whereas the convolution integral accounts for the retarded friction due to correlated sequences of collisions. A short-time expansion of the e.o.m, Eq. (8), yields $T_{s}^{\perp}(t) / T_{s}^{\perp}(0)=1-t / \tau+\mathcal{O}\left(t^{2}\right)$, and the convolution integral over the memory kernel $K_{s}^{\perp}(t)$ contributes only to order $\mathcal{O}\left(t^{2}\right)$. Direct expansion of $\delta \epsilon_{s}^{\perp}(t)=\exp \left(i \mathcal{L}_{ \pm} t\right) \delta \epsilon_{s}^{\perp}$ in powers of $t$ in $T_{s}^{\perp}(t)$ yields for the relaxation rate the microscopic expression

$$
\tau^{-1}=\left\langle\delta \epsilon_{s}^{\perp}\left(\mp i \mathcal{L}_{ \pm}^{\text {int }} \delta \epsilon_{s}^{\perp}\right)\right\rangle \frac{2}{\left(k_{B} T\right)^{2}} .
$$

For the memory kernel a microscopic expression also follows

$$
K_{s}^{\perp}(t)=\left\langle\mathcal{Q} \mathcal{L}_{+}^{\mathrm{int}} \delta \epsilon_{s}^{\perp}\left|e^{-i \mathcal{Q} \mathcal{L}_{-} \mathcal{Q} t}\right| \mathcal{Q} \mathcal{L}_{-}^{\mathrm{int}} \delta \epsilon_{s}^{\perp}\right\rangle \frac{2}{\left(k_{B} T\right)^{2}},
$$


where $\mathcal{Q}$ projects onto the subspace orthogonal to $\delta \epsilon_{s}^{\perp}$ and the bracket corresponds to Kubo's scalar product [52-54]. Since the memory kernel contains at least two operators $\mathcal{L}_{ \pm}^{\text {int }}$ which we identified as small perturbation, the retarded convolution term in Eq. (8) becomes negligible with respect to the instantaneous relaxation. Then the e.o.m. [Eq. (8)] simplifies to an exponential relaxation as leading behavior consistent with our simulation results.

The relaxation rate, Eq. (9), involves an equilibrium average of essentially the collision operator. Its direct evaluation becomes feasible for strong confinement relying on the decoupling property of the ensemble into lateral and transversal degrees of freedom, as well as the usual decoupling of the structural and kinetic degrees of freedom. The second crucial ingredient is that the unit vector $\mathbf{e}=\left(\mathbf{e}^{\|}, e^{\perp}\right)$ for the contact condition becomes more and more confined to the planar direction, $\left|e^{\perp}\right| \leq L / \sigma \ll 1$. By the collision rule, Eq. (5), $\tilde{v}_{s}^{\perp}=v_{s}^{\perp}+e^{\perp}\left(\mathbf{e} \cdot \mathbf{v}_{m s}\right)$ the transverse velocity remains almost unchanged after a collision. After performing the structural and kinetic averages we obtain as leading contribution (see Supplemental Material [54])

$$
\tau^{-1}=\frac{16 \varphi_{2 D}}{3 \sqrt{\pi}}\left(\frac{L}{\sigma}\right)^{2} g(\sigma) t_{0}^{-1}
$$

where $g(\sigma)$ the radial distribution function of the twodimensional hard-disk fluid at contact. The factor $\varphi_{2 D} g(\sigma)$ accounts for the probability of a scattering event similar to Enskog's theory for bulk hard-sphere fluid [52]. In contrast to the $3 \mathrm{D}$ case here it arises as an exact result valid at any packing fraction $\varphi_{2 D}$. The decoupling of the lateral and transversal degrees of freedom is encoded in the factor $(L / \sigma)^{2}$ reflecting the small momentum transfer in quasi-planar collisions.

The radial distribution function $g(r)$ can be evaluated within integral equations theory [52]. Here we rely on a numerical solution of the 2D Percus-Yevick closure relation [60] that compares quantitatively to our measured $g(r)$ in the simulation, see Fig. 4. Using the contact value $g(\sigma)$ the prefactor $A\left(\varphi_{2 D}\right)$ for the relaxation rate in Eq. (2) can be compared to the analytic result, Eq. (11). The comparison in Fig. 4 for low to moderate packing fractions $\varphi_{2 D}$ corroborates that the theoretical prediction is in fact an exact result.

Conclusions We have demonstrated the emergence of a divergent time scale for the coupling of lateral to transverse degrees of freedom in a strongly confined fluid. The main insight has been that in collisions the momentum transfer becomes more and more planar as the wall separation is reduced. The dependence of the divergent time scale on the plate separation and packing fraction of the fluid has been worked out analytically including the prefactor by evaluating the dominant contribution of the collision operator and compared to our simulations.
Remarkably, the theory is not limited to fluids but also applies to the ordered phase. We emphasize that the reference system is strongly interacting and our calculation is one of the rare cases where analytic results can be elaborated.

The mechanism unraveled for the emergence of a slow time scale and the scaling with the transverse dimension should also hold for other geometries such as liquids in narrow cylindrical tubes or quasi-1D confinement (see Supplemental Material [54]).

The hard-core interaction is of course an idealization of a short-ranged potential, but we anticipate our results to remain valid for the case of smooth potentials. More precisely, for hard spheres the collisions are instantaneous whereas for smooth potentials the duration of a collision introduces a new time scale into the problem. As long as the Knudsen time scale $L / v_{t h}$, i.e. the typical time for a particle to traverse the slit, is still much larger than the duration of a collision the mechanisms for small transverse momentum transfer should be identical (see Supplementary Material for simulation results on smooth potentials [54]). Similarly, a smooth particlewall interaction should not modify our findings, provided its range is much smaller than the slit width, and the transverse energy includes the wall potential in addition to the transverse kinetic energy.

It is also of interest to consider the opposite case where the duration of a collision is much longer than the time to traverse the slit. Then the use of a collision operator is no longer justified; rather, the collision events can be averaged over the fast transverse oscillations. Analytic progress in this direction has been made very recently [61] and it turns out that the predicted relaxation time for this case scales with a different power in the wall separation. Furthermore, the relaxation of the kinetic energy becomes exponential at times much longer than the Knudsen time, while for hard spheres it is exponential for all times.

The diverging relaxation time separates the decoupled two-dimensional dynamics from the coupled one in strong confinement. This should have drastic implications for systems in the vicinity of the glass transition such that the divergent structural relaxation time competes with the relaxation time of the coupling. In fact the modecoupling theory for confinement [47] suggests that the limits $t \rightarrow \infty$ and $L \rightarrow 0$ do not commute and different glassy dynamics on different time scales is expected.

The decoupling property of the transverse and lateral degrees of freedom in the equilibrium ensemble implies a divergent time scale for their dynamic coupling. The precise form of the divergence should depend on the microdynamics and should be different for the case of Brownian dynamics, which can be realized experimentally for colloids confined between glass plates. Yet, to measure the divergent time scale in this case an observable needs to be chosen that does not relax quickly to 
equilibrium even without the close-to-planar collisions. An example could be the in-plane self-intermediate scattering function $F^{s}(q, t)$ at small wave numbers $q$, which probes the planar dynamics at large lateral length scales $2 \pi / q$. Upon decreasing the wavenumber the relaxation time slows down as $\sim q^{-2}$ by diffusion and the crossover from purely $2 \mathrm{D}$ motion to the $3 \mathrm{D}$ confined coupled dynamics should be visible. A second, more challenging candidate for such an observable is the generalized intermediate scattering functions for fluids close to the glass transition.

We gratefully acknowledge many discussions with Rolf Schilling on our simulation results and on the emergence of the diverging time scale. This work has been supported by the Deutsche Forschungsgemeinschaft DFG via the Research Unit FOR1394 "Nonlinear Response to Probe Vitrification".

[1] D. P. Sheehan, Phys. Rev. E 88, 032125 (2013).

[2] M. Sahimi, Rev. Mod. Phys. 65, 1393 (1993).

[3] S. Granick, Science 253, 1374 (1991).

[4] A. Z. Szeri, Fluid Film Lubrication, 2nd ed. (Cambridge University Press, 2010).

[5] J. Santana-Solano, A. Ramírez-Saito, and J. L. ArauzLara, Phys. Rev. Lett. 95, 198301 (2005).

[6] M. L. Ekiel-Jeżewska, E. Wajnryb, J. Bławzdziewicz, and F. Feuillebois, J. Chem. Phys. 129, 181102 (2008).

[7] N. L. Rosi, J. Eckert, M. Eddaoudi, D. T. Vodak, J. Kim, M. O'Keefe, and O. M. Yaghi, Science 300, 1127 (2003).

[8] H. S. Quah, W. Chen, M. K. Schreyer, H. Yang, M. W. Wong, W. Ji, and J. J. Vittal, Nat. Commun. 6, 7954 (2015).

[9] T. Tomita, K. Nakayama, and H. Sakai, Microporus and Mesoporus Materials 68, 71 (2004).

[10] J. Kärger, D. M. Ruthven, and D. N. Theodorou, Diffusion in nanoporous materials (John Wiley \& Sons, 2012).

[11] S. Wang, T. J. Kowal, M. K. Marei, M. M. Falk, and H. Jain, Tissue Engineering Part A 19, 1632 (2013).

[12] L. Schlapbach and A. Züttel, Nature 414, 353 (2001).

[13] J. Han, J. Fu, and R. B. Schoch, Lab Chip 8, 23 (2008).

[14] J. C. T. Eijkel and A. v. d. Berg, Microfluidics and Nanofluidics 1, 249 (2005).

[15] D. Mijatovic, J. C. T. Eijkel, and A. van den Berg, Lab Chip 5, 492 (2005).

[16] D.-H. Lien, J. R. D. Retamal, J.-J. Ke, C.-F. Kang, and J.-H. He, Nanoscale 7, 19874 (2015).

[17] M. Knudsen, Ann. Phys. 333 (1909).

[18] M. v. Smoluchowski, Ann. Phys. 338 (1910).

[19] G. Arya, H.-C. Chang, and E. J. Maginn, Phys. Rev. Lett. 91, 026102 (2003).

[20] S. Gruener and P. Huber, Phys. Rev. Lett. 100, 064502 (2008).

[21] K. Malek and M.-O. Coppens, Phys. Rev. Lett. 87, 125505 (2001).

[22] H. Kim, C. Kim, E. K. Lee, P. Talkner, and P. Hänggi, Phys. Rev. E 77, 031202 (2008).

[23] H. Löwen, J. Phys.: Condens. Matter 21, 474203 (2009).

[24] N. Saklayen, G. L. Hunter, K. V. Edmond, and E. R.
Weeks, AIP Conf. Proc. 1518 (2013).

[25] G. L. Hunter, K. V. Edmond, and E. R. Weeks, Phys. Rev. Lett. 112, 218302 (2014).

[26] B. Zhang and X. Cheng, Phys. Rev. Lett. 116, 098302 (2016).

[27] M. Schmidt and H. Löwen, Phys. Rev. Lett. 76, 4552 (1996); Phys. Rev. E 55, 7228 (1997).

[28] N. Gribova, A. Arnold, T. Schilling, and C. Holm, J. Chem. Phys. 135, 054514 (2011).

[29] A. Fortini and M. Dijkstra, J. Phys. Condens. Matter 18, L371 (2006).

[30] A. B. Fontecha, H. J. Schöpe, H. König, T. Palberg, R. Messina, and H. Löwen, J. Phys. Condens. Matter 17, S2779 (2005).

[31] E. C. Oğuz, R. Messina, and H. Löwen, Europhys. Lett. 86, 28002 (2009); E. C. Oğuz, A. Reinmüller, H. J. Schöpe, T. Palberg, R. Messina, and H. Löwen, J. Phys.: Condens. Matter 24, 464123 (2012); E. C. Oğuz, M. Marechal, F. Ramiro-Manzano, I. Rodriguez, R. Messina, F. J. Meseguer, and H. Löwen, Phys. Rev. Lett. 109, 218301 (2012).

[32] A. Reinmüller, T. Palberg, and H. J. Schöpe, Rev. Sci. Instrum. 84, 063907 (2013).

[33] F. Varnik and T. Franosch, Journal of Physics: Condensed Matter 28, 133001 (2016).

[34] C. Alba-Simionesco, B. Coasne, G. Dosseh, G. Dudziak, K. Gubbins, R. Radhakrishnan, and M. SliwinskaBartkowiak, Journal of Physics: Condensed Matter 18, R15 (2006).

[35] W. Qi, Y. Peng, Y. Han, R. K. Bowles, and M. Dijkstra, Phys. Rev. Lett. 115, 185701 (2015).

[36] B. Li, D. Zhou, and Y. Han, Nature Reviews Materials 1, 15011 (2016).

[37] J. Klein and E. Kumacheva, Science 269, 816 (1995); J. Chem. Phys. 108, 6996 (1998); E. Kumacheva and J. Klein, J. Chem. Phys. 108, 7010 (1998).

[38] D. F. Kienle and T. L. Kuhl, Phys. Rev. Lett. 117, 036101 (2016).

[39] A. L. Demirel and S. Granick, J. Chem. Phys. 115, 1498 (2001).

[40] Y. Zhu and S. Granick, Phys. Rev. Lett. 93, 096101 (2004).

[41] C. R. Nugent, K. V. Edmond, H. N. Patel, and E. R. Weeks, Phys. Rev. Lett. 99, 025702 (2007); K. Nygård, R. Kjellander, S. Sarman, S. Chodankar, E. Perret, J. Buitenhuis, and J. F. van der Veen, Phys. Rev. Lett. 108, 037802 (2012); K. Nygård, S. Sarman, K. Hyltegren, S. Chodankar, E. Perret, J. Buitenhuis, J. F. van der Veen, and R. Kjellander, Phys. Rev. X 6, 011014 (2016); K. Nygård, J. Buitenhuis, M. Kagias, K. Jefimovs, F. Zontone, and Y. Chushkin, Phys. Rev. Lett. 116, 167801 (2016).

[42] D. K. Satapathy, O. Bunk, K. Jefimovs, K. Nygård, H. Guo, A. Diaz, E. Perret, F. Pfeiffer, C. David, G. H. Wegdam, and J. F. van der Veen, Phys. Rev. Lett. 101, 136103 (2008); D. K. Satapathy, K. Nygård, O. Bunk, K. Jefimovs, E. Perret, A. Diaz, F. Pfeiffer, C. David, and J. F. van der Veen, EPL (Europhysics Letters) 87, 34001 (2009).

[43] J. Mittal, J. R. Errington, and T. M. Truskett, Phys. Rev. Lett. 96, 177804 (2006); J. Phys. Chem. B 111, 10054 (2007); J. Chem. Phys. 126, 244708 (2007); J. Mittal, T. M. Truskett, J. R. Errington, and G. Hummer, Phys. Rev. Lett. 100, 145901 (2008). 
[44] T. S. Ingebrigtsen, J. R. Errington, T. M. Truskett, and J. C. Dyre, Phys. Rev. Lett. 111, 235901 (2013).

[45] G. Goel, W. P. Krekelberg, J. R. Errington, and T. M. Truskett, Phys. Rev. Lett. 100, 106001 (2008); G. Goel, W. P. Krekelberg, M. J. Pond, J. Mittal, V. K. Shen, J. R. Errington, and T. M. Truskett, J. Stat. Mech. Theo. Exp. 2009, P04006 (2009); W. P. Krekelberg, D. W. Siderius, V. K. Shen, T. M. Truskett, and J. R. Errington, Langmuir 29, 14527 (2013).

[46] T. Fehr and H. Löwen, Phys. Rev. E 52, 4016 (1995).

[47] S. Lang, V. Boţan, M. Oettel, D. Hajnal, T. Franosch, and R. Schilling, Phys. Rev. Lett. 105, 125701 (2010); S. Lang, R. Schilling, V. Krakoviack, and T. Franosch, Phys. Rev. E 86, 021502 (2012); S. Lang, R. Schilling, and T. Franosch, J. Stat. Mech. 12, P12007 (2013); Phys. Rev. E 90, 062126 (2014).

[48] S. Mandal, S. Lang, M. Gross, M. Oettel, D. Raabe, T. Franosch, and F. Varnik, Nat. Commun. 5, 5435 (2014).

[49] T. Franosch, S. Lang, and R. Schilling, Phys. Rev. Lett. 109, 240601 (2012); S. Lang, T. Franosch, and R. Schilling, J. Chem. Phys. 140, 104506 (2014).

[50] M. Ernst, J. Dorfman, W. Hoegy, and J. V. Leeuwen,
Physica 45, 127 (1969).

[51] N. V. Brilliantov and T. Pöschel, Kinetic theory of granular gases (Oxford University Press, 2010).

[52] J. P. Hansen and I. R. McDonald, Theory of Simple Liquids (Academic Press, 2006).

[53] W. Götze, Complex Dynamics of Glass-Forming LiquidsA Mode-Coupling Theory (Oxford University, Oxford, 2009).

[54] See Supplemental Material which includes Ref. [55-59], for details on the Zwanzig-Mori equations and smooth potentials.

[55] J. A. Bollinger, J. Carmer, A. Jain, and T. M. Truskett, Soft Matter 12, 9561 (2016).

[56] J. A. Bollinger, A. Jain, J. Carmer, and T. M. Truskett, J. Chem. Phys. 142, 161102 (2015).

[57] J. D. Weeks, D. Chandler, and H. C. Andersen, J. Chem. Phys. 54, 5237 (1971).

[58] S. Plimpton, J. Comput. Phys. 117, 1 (1995).

[59] L. Tonks, Phys. Rev. 50, 955 (1936).

[60] M. Adda-Bedia, E. Katzav, and D. Vella, J. Chem. Phys. 128, 184508 (2008).

[61] R. Schilling, Phys. Rev. E 93, 062102 (2016). 


\section{SUPPLEMENTAL MATERIAL}

\section{ZWANZIG-MORI EQUATIONS OF MOTION AND RELAXATION TIME}

Consider the fluctuations of the transverse kinetic energy of a a tagged particle

$$
\delta \epsilon_{s}^{\perp}:=\frac{m}{2}\left(v_{s}^{\perp}\right)^{2}-\frac{1}{2} k_{B} T,
$$

where the label $s$ is any of the $1, \ldots, N$ and $v_{s}^{\perp}$ the velocity component perpendicular to the walls, $m$ the mass of the particle and $k_{B} T$ the thermal energy. By equipartion $\left\langle\delta \epsilon_{s}^{\perp}\right\rangle=0$. In the limit of small wall separation the ensemble decouples and $\delta \epsilon_{s}^{\perp}$ becomes a conserved quantity. Here we derive an exact equation of motion for the time-correlation function of $\epsilon_{s}^{\perp}(t)=e^{i \mathcal{L}_{ \pm} t} \epsilon_{s}^{\perp}$

$$
T_{s}^{\perp}(t):=\left\langle\delta \epsilon_{s}^{\perp}(t) \delta \epsilon_{s}^{\perp}\right\rangle=\left\langle e^{i \mathcal{L}_{ \pm} t} \delta \epsilon_{s}^{\perp} \mid \delta \epsilon_{s}^{\perp}\right\rangle=\left\langle\delta \epsilon_{s}^{\perp} \mid e^{-i \mathcal{L}_{\mp} t} \delta \epsilon_{s}^{\perp}\right\rangle, \quad t \gtrless 0 .
$$

Here $\langle A \mid B\rangle \equiv\left\langle A^{*} B\right\rangle$ abbreviates the Kubo scalar product [53] and the adjoint of the pseudo-Liouvillian with respect to this scalar product fulfills $\left(\mathcal{L}_{ \pm}\right)^{\dagger}=\mathcal{L}_{ \pm}$. In the following we restrict the discussion to non-negative times $t \geq 0$ only, negative times follow trivially. The initial value is readily calculated since the momenta are drawn from a Maxwell-Boltzmann distribution

$$
T_{s}^{\perp}(t=0)=\left\langle\left|\delta \epsilon_{s}^{\perp}\right|^{2}\right\rangle=\frac{1}{2}\left(k_{B} T\right)^{2} .
$$

We rely on the operator identity for the backwards-time evolution operator $\mathcal{R}(t)=\exp \left(-i \mathcal{L}_{\mp} t\right), t \gtrless 0$

$$
\partial_{t} \mathcal{P} \mathcal{R}(t) \mathcal{P}+i \mathcal{P} \mathcal{L}_{\mp} \mathcal{P} \mathcal{R}(t) \mathcal{P}+\int_{0}^{t} \mathrm{~d} t^{\prime} \mathcal{P} \mathcal{L}_{\mp} \mathcal{Q} \mathcal{R}_{\mathcal{Q}}\left(t-t^{\prime}\right) \mathcal{Q} \mathcal{L}_{\mp} \mathcal{P} \mathcal{R}(t) \mathcal{P}=0
$$

valid for any orthogonal projector $\mathcal{P}$, see e.g. [47]. Here $\mathcal{Q}=\mathbf{1}-\mathcal{P}$ denotes the projection onto the orthogonal complement, and $\mathcal{R}_{\mathcal{Q}}(t)=\exp \left(-i \mathcal{Q} \mathcal{L}_{\mp} \mathcal{Q} t\right)$ is referred to as the reducd backwards-time evolution operator.

For the projector we choose

$$
\mathcal{P}=\left|\delta \epsilon_{s}^{\perp}\right\rangle \frac{2}{\left(k_{B} T\right)^{2}}\left\langle\delta \epsilon_{s}^{\perp}\right|,
$$

which by the normalization Eq. (14) indeed fulfills $\mathcal{P}=\mathcal{P}^{2}=\mathcal{P}^{\dagger}$. From the operator identity, Eq. (15) one finds the exact Zwanzig-Mori equation of motion

$$
\dot{T}_{s}^{\perp}(t) \pm \tau^{-1} T_{s}^{\perp}(t)+\int_{0}^{t} K_{s}^{\perp}\left(t-t^{\prime}\right) T_{s}^{\perp}\left(t^{\prime}\right) \mathrm{d} t^{\prime}=0, \quad t \gtrless 0
$$

An explicit expression for the characteristic relaxation rate $\tau^{-1}$ follows

$$
\tau^{-1}=\left\langle\delta \epsilon_{s}^{\perp} \mid \pm i \mathcal{L}_{\mp}^{\mathrm{int}} \delta \epsilon_{s}^{\perp}\right\rangle \frac{2}{\left(k_{B} T\right)^{2}},
$$

and similarly for the memory kernel

$$
K_{s}^{\perp}(t)=\left\langle\mathcal{Q} \mathcal{L}_{ \pm}^{\text {int }} \delta \epsilon_{s}^{\perp}\left|e^{-i \mathcal{Q} \mathcal{L}_{\mp} \mathcal{Q} t}\right| \mathcal{Q} \mathcal{L}_{\mp}^{\text {int }} \delta \epsilon_{s}^{\perp}\right\rangle \frac{2}{\left(k_{B} T\right)^{2}}, \quad t \gtrless 0,
$$

where we observed $\mathcal{L}_{ \pm} \delta \epsilon_{s}^{\perp}=\mathcal{L}_{ \pm}^{\text {int }} \epsilon_{s}^{\perp}$.

Here we focus on the evaluation of the relaxation rate $\tau^{-1}$ of the transeverse kinetic energy, Eq. (18). Since the transverse dynamics conserves the transverse kinetic energy and $\delta \epsilon_{s}^{\perp}$ does not involve lateral degrees of freedom, only collisions contribute to the relevant matrix element

$$
\begin{aligned}
& \left\langle\delta \epsilon_{s}^{\perp} \mid i \mathcal{L}_{-}^{\mathrm{int}} \delta \epsilon_{s}^{\perp}\right\rangle=\left\langle\delta \epsilon_{s}^{\perp} \mid \sum_{m=1, m \neq s}^{N} \delta \hat{T}_{-}(m, s) \delta \epsilon_{s}^{\perp}\right\rangle \\
= & \sum_{\substack{m=1 \\
m \neq s}}^{N}\left\langle\sigma^{2} \int \mathrm{d} \mathbf{e} \Theta\left(\mathbf{v}_{m s} \cdot \mathbf{e}\right)\left|\mathbf{v}_{m s} \cdot \mathbf{e}\right|\left[\delta^{(3)}\left(\mathbf{x}_{m s}-\sigma \mathbf{e}\right)-\delta^{(2)}\left(\mathbf{x}_{m s}^{\|}-\sigma \mathbf{e}^{\|}\right) \delta\left(\sigma e^{\perp}\right)\right]\left[\frac{m}{2}\left(v_{s}^{\perp}\right)^{2}-\frac{1}{2} k_{B} T\right]\left[\frac{m}{2}\left(\tilde{v}_{s}^{\perp}\right)^{2}-\frac{m}{2}\left(v_{s}^{\perp}\right)^{2}\right]\right\rangle,
\end{aligned}
$$


with the post-collision velocity $\tilde{\mathbf{v}}_{s}=\left(\tilde{\mathbf{v}}_{s}^{\|}, \tilde{v}_{s}^{\perp}\right)=\mathbf{v}_{s}+\mathbf{e}\left(\mathbf{e} \cdot \mathbf{v}_{m s}\right)$ of the tagged particle. Since for purely planar collisions the transverse velocity does not change the second $\delta$-functions does not contribute.

For small wall separations, Fig. 1 of the main text reveals that the unit vector $\mathbf{e}=\left(\mathbf{e}^{\|}, e^{\perp}\right)$ is confined to a small region around the equator $\left|e^{\perp}\right| \leq L / \sigma \ll 1,\left|\mathbf{e}^{\|}\right| \simeq 1$. Furthermore the thermal average over the structural degrees of freedom can be performed observing that in the reference ensemble the degrees of freedom decouple into a two-dimensional interacting fluid for the lateral degrees of freedom and an ideal gas for the transverse ones [49].

$$
\begin{aligned}
\left\langle\delta \epsilon_{s}^{\perp} \mid i \mathcal{L}_{-}^{\text {int }} \delta \epsilon_{s}^{\perp}\right\rangle= & n_{0} \sigma^{2} g(\sigma) \int \mathrm{d} \mathbf{e}\left\langle\Theta\left(\mathbf{v}_{m s} \cdot \mathbf{e}\right)\left|\mathbf{v}_{m s} \cdot \mathbf{e}\right|\left[\frac{m}{2}\left(v_{s}^{\perp}\right)^{2}-\frac{1}{2} k_{B} T\right]\left[\frac{m}{2}\left(\tilde{v}_{s}^{\perp}\right)^{2}-\frac{m}{2}\left(v_{s}^{\perp}\right)^{2}\right]\right\rangle \\
& \times \int \frac{\mathrm{d} z_{m}}{L} \int \frac{\mathrm{d} z_{s}}{L} \delta\left(z_{m}-z_{s}-\sigma e^{\perp}\right) .
\end{aligned}
$$

Here $n_{0}=N / A$ is the two-dimensional particle density and $g(\sigma)$ the radial distribution function of the two-dimensional reference fluid at contact. The integral in the preceeding equations over the transverse degrees of freedom extends from $-L / 2$ to $L / 2$. By a change of variables $z_{m s}=z_{m}-z_{s}$

$$
\begin{aligned}
\int_{-L / 2}^{L / 2} \mathrm{~d} z_{m} \int_{-L / 2}^{L / 2} \mathrm{~d} z_{s} \delta\left(z_{m}-z_{s}-\sigma e^{\perp}\right) & =\int_{-L}^{L} \mathrm{~d} z_{m s} \delta\left(z_{m s}-\sigma e^{\perp}\right) \int_{z_{s} \in[-L / 2, L / 2], z_{s}+z_{m s} \in[-L / 2, L / 2]} \mathrm{d} z_{s} \\
& =\int_{-L}^{L} \mathrm{~d} z_{m s} \delta\left(z_{m s}-\sigma e^{\perp}\right)\left(L-\left|z_{m s}\right|\right) \\
& =L-\sigma\left|e^{\perp}\right|
\end{aligned}
$$

and we understand that from now on $\left|e^{\perp}\right| \leq L / \sigma$. The integral over the orientation of the contact point thus collapses to a small margin at the equator:

$$
\int \mathrm{d} \mathbf{e} \Theta\left(\mathbf{v}_{m s} \cdot \mathbf{e}\right) \ldots \mapsto \int_{-L / \sigma}^{L / \sigma} \mathrm{d} e^{\perp} \int \mathrm{d} \mathbf{e}^{\|} \Theta\left(\mathbf{v}_{m s}^{\|} \cdot \mathbf{e}^{\|}\right) \ldots
$$

where $e^{\perp}$ is the latitude on the unit sphere and the integral de $\mathbf{e}^{\|}$extends over a unit circle. Then the matrix element further simplifies to

$$
\begin{aligned}
& \left\langle\delta \epsilon_{s}^{\perp} \mid i \mathcal{L}_{-}^{\mathrm{int}} \delta \epsilon_{s}^{\perp}\right\rangle= \\
& =n_{0} \sigma g(\sigma) \int_{-L / \sigma}^{L / \sigma}\left(1-\frac{\sigma}{L}\left|e^{\perp}\right|\right) \frac{\sigma}{L} \mathrm{~d} e^{\perp} \int \mathrm{d} \mathbf{e}^{\|}\left\langle\Theta\left(\mathbf{v}_{m s}^{\|} \cdot \mathbf{e}^{\|}\right)\left(\mathbf{v}_{m s} \cdot \mathbf{e}\right)\left[\frac{m}{2}\left(v_{s}^{\perp}\right)^{2}-\frac{1}{2} k_{B} T\right]\left[\frac{m}{2}\left(\tilde{v}_{s}^{\perp}\right)^{2}-\frac{m}{2}\left(v_{s}^{\perp}\right)^{2}\right]\right\rangle .
\end{aligned}
$$

After integrating over the latitude $e^{\perp}$ only even powers in the thermal average $\langle\ldots\rangle$ contribute. Furthermore since the contact point are close to the equator we need only the lowest even contribution in $e^{\perp}$. Using the collision rule

$$
\tilde{v}_{s}^{\perp}=v_{s}^{\perp}+e^{\perp}\left(\mathbf{v}_{m s} \cdot \mathbf{e}\right)=v_{s}^{\perp}+e^{\perp}\left(\mathbf{v}_{m s}^{\|} \cdot \mathbf{e}^{\|}\right)+e^{\perp} v_{m s}^{\perp} e^{\perp}
$$

the thermal average in the bracket of Eq. (24) reduces to (keeping in each step only the even leading terms in $e^{\perp}$ )

$$
\begin{aligned}
\langle\ldots\rangle & =\left\langle\Theta\left(\mathbf{v}_{m s}^{\|} \cdot \mathbf{e}^{\|}\right)\left[\frac{m}{2}\left(v_{s}^{\perp}\right)^{2}-\frac{1}{2} k_{B} T\right] \frac{m}{2}\left[2 v_{s}^{\perp} e^{\perp}\left(\mathbf{v}_{m s} \cdot \mathbf{e}\right)^{2}+\left(e^{\perp}\right)^{2}\left(\mathbf{v}_{m s} \cdot \mathbf{e}\right)^{3}\right]\right\rangle \\
& =\left\langle\Theta\left(\mathbf{v}_{m s}^{\|} \cdot \mathbf{e}^{\|}\right)\left[\frac{m}{2}\left(v_{s}^{\perp}\right)^{2}-\frac{1}{2} k_{B} T\right] \frac{m}{2}\left[4\left(v_{s}^{\perp} e^{\perp}\right)^{2}\left(\mathbf{v}_{m s}^{\|} \cdot \mathbf{e}^{\|}\right)+\left(e^{\perp}\right)^{2}\left(\mathbf{v}_{m s}^{\|} \cdot \mathbf{e}^{\|}\right)^{3}\right]\right\rangle,
\end{aligned}
$$

since $v_{s}^{\perp}$ is a gaussian variable the second term in the second bracket also does not contribute, and the remaining average factorizes into gaussian integrals

$$
\begin{aligned}
\langle\ldots\rangle & =4\left(e^{\perp}\right)^{2}\left\langle\Theta\left(\mathbf{v}_{m s}^{\|} \cdot \mathbf{e}^{\|}\right) \mathbf{v}_{m s}^{\|} \cdot \mathbf{e}^{\|}\right\rangle\left\langle\left[\frac{m}{2}\left(v_{s}^{\perp}\right)^{2}-\frac{1}{2} k_{B} T\right] \frac{m}{2}\left(v_{s}^{\perp}\right)^{2}\right\rangle \\
& =\left(e^{\perp}\right)^{2} \frac{2 v_{t h}}{\sqrt{\pi}}\left(k_{B} T\right)^{2} .
\end{aligned}
$$


Here we used the elementary result $\left\langle\left(\mathbf{v}_{m s}^{\|} \cdot \mathbf{e}^{\|}\right) \Theta\left(\mathbf{v}_{m s}^{\|} \cdot \mathbf{e}^{\|}\right\rangle=v_{t h} / \sqrt{\pi}\right.$ for the average relative velocity along the approaching direction $\mathbf{e}^{\|}$. With

$$
\int_{-L / \sigma}^{L / \sigma} \frac{\sigma}{L} \mathrm{~d} e^{\perp}\left(e^{\perp}\right)^{2}\left(1-\frac{\sigma}{L}\left|e^{\perp}\right|\right)=\frac{L^{2}}{6 \sigma^{2}}
$$

the remaining integrals in Eq. (24) now yield

$$
\left\langle\delta \epsilon_{s}^{\perp} \mid i \mathcal{L}_{-}^{\mathrm{int}} \delta \epsilon_{s}^{\perp}\right\rangle=\frac{2 n_{0}}{3 \sigma} L^{2} g(\sigma)\left(k_{B} T\right)^{2} v_{t h} \sqrt{\pi} .
$$

For the relaxation rate in Eq. (18) this implies the principal result of this work

$$
\tau^{-1}=\frac{16 \varphi_{2 \mathrm{D}}}{3 \sqrt{\pi}}\left(\frac{L}{\sigma}\right)^{2} g(\sigma) t_{0}^{-1} .
$$

where we reintroduced the two-dimensional packing fraction $\varphi_{2 \mathrm{D}}=n_{0} \pi \sigma^{2} / 4$ and the natural time scale $t_{0}=\sigma / v_{t h}$

\section{SMOOTH POTENTIALS}
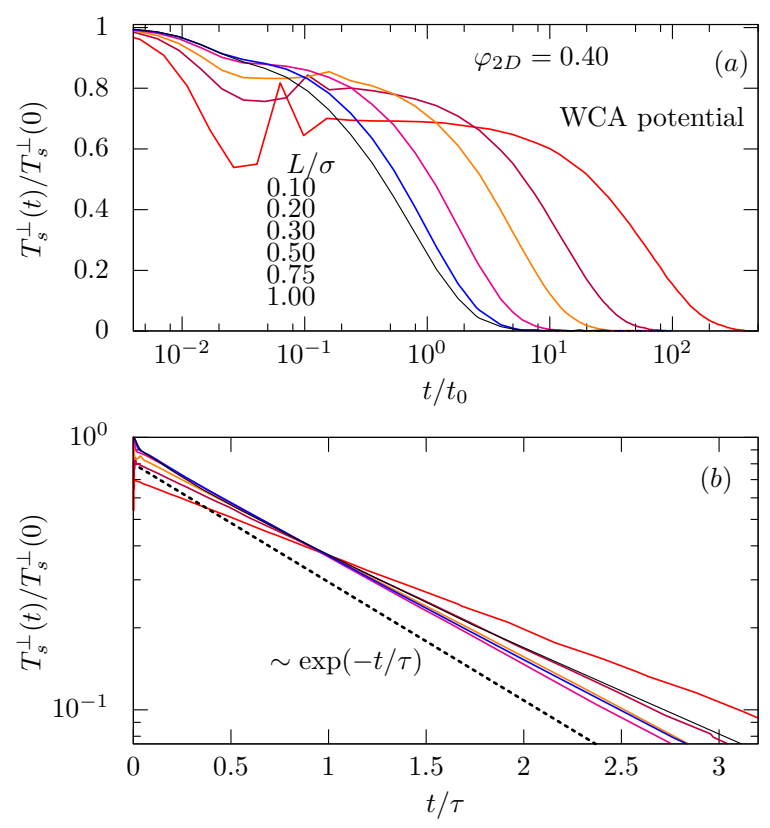

FIG. 5. Decay of the time-correlation function $T_{s}^{\perp}(t)$ of the transversal kinetic energy of a liquid with smooth interactions for packing fraction $\varphi_{2 D}=0.40$ and various wall separations $L$. Wall distance decreases from left to right. (b) Same data in semi-log plot after rescaling with the measured relaxation time $\tau$.

We further simulate particles using a steep Weeks-Chandler-Andersen (WCA) potential as used in Ref. [55, 56], which captures many properties of dense atomistic liquids [57]. The particle-particle interactions are defined as $u_{p p}(r)=4 \epsilon\left[(\sigma / r)^{48}-(\sigma / r)^{24}\right]+\epsilon$ for $r \leq 2^{1 / 24} \sigma$ and $u_{p p}(r)=0$ for $r \geq 2^{1 / 24} \sigma$, where $r$ and $\sigma$ are the inter-particle separation and particle diameter, respectively. For convenience, we use the characteristic energy scale $\epsilon=k_{B} T$, which sets the time scale $t_{0}=\sqrt{m \sigma^{2} / \epsilon}$. This liquid is confined to a slit between two parallel and flat walls placed at $\pm(\sigma+L / 2)$ by using a steep WCA interactions, such that the accessible slit width is still given by $L$. Particles located inside the slit interact with both walls, we employ the wall-particle interaction $u_{w p}(z)=4 \epsilon\left[(\sigma / z)^{48}-(\sigma / z)^{24}\right]+\epsilon$ for $z \leq 2^{1 / 24} \sigma$ and $u_{w p}(z)=0$ for $z \geq 2^{1 / 24} \sigma$, where $z$ is the distance to one of the walls. We have performed NVE molecular dynamics simulations using LAMMPS [58], and integrate Newton's equations of motion with a time step of $\delta t=10^{-5} t_{0}$. We equilibriate the systems for sufficintly long time such that on average each particle moves more than $10 \sigma$. 
quasi-2D

In order to quantify the divergent time scale, we calculate the time-correlation function $T_{s}^{\perp}(t)=\left\langle\delta \epsilon_{s}^{\perp}(t) \delta \epsilon_{s}^{\perp}(0)\right\rangle$ as shown in Fig. 5. Since the potential is very steep it does not contribute significantly to the total transverse energy. One observes that the characteristic time scale increases by 2 orders of magnitude as the plate separation is decreased by a factor of 10. In contrast to hard sphere systems, these time-correlation functions are not purely exponential at very short time scales, which reflect that the collisions are no longer instantaneous. Yet, plotting the data on a semi-logarithmic plot reveals that the relaxation function becomes exponential at times much larger than the collision time.

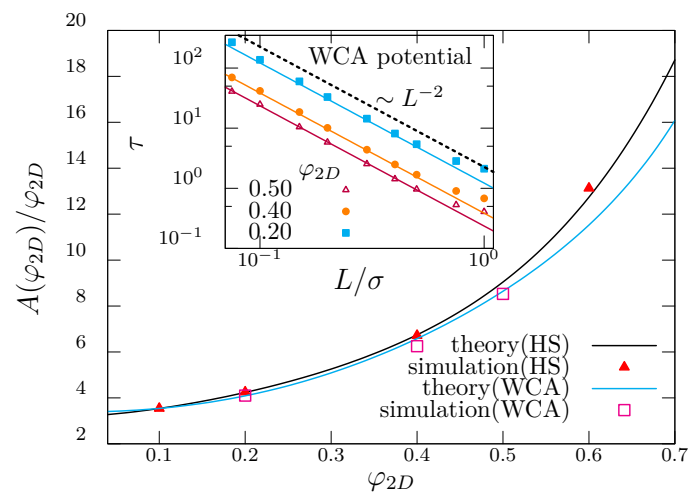

FIG. 6. Prefactor $A\left(\varphi_{2 \mathrm{D}}\right)$ for both the hard sphere system and the WCA liquid extracted from the scaling behavior of the relaxation time $\tau\left(L, \varphi_{2 D}\right)^{-1}=t_{0}^{-1}(L / \sigma)^{2} A\left(\varphi_{2 D}\right)$ as a function of $2 D$ packing fraction $\varphi_{2 D}$. The inset shows the relaxation time $\tau$ for the strongly confined WCA liquids as a function of the wall separation $L$ for various packing fractions $\varphi_{2 D}$. Packing fraction increases from top to bottom. The dashed line is a power law $\sim L^{-2}$ and serves as a guide to the eye.

The relaxation time $\tau=\tau\left(L, \varphi_{2 \mathrm{D}}\right)$ extracted from the WCA simulations, see the inset of Fig. 6, approaches the scaling law $\sim L^{-2}$ for all packing fractions considered. For comparison with Eq. (31) we use the maximum of the pair-distribution function $g(r)$ to replace the contact value $g(\sigma)$ in the case of hard spheres. The prefactor of the scaling law is shown Fig. 6 for hard spheres and the WCA liquid for various densities and is described nicely by the theory. Last, we have also tuned the particle-particle interactions of the WCA potential by changing the pair of exponents from $(48-24)$ to $(24-12)$, but still observe the same divergence of the time scale $\sim L^{-2}$.

\section{quasi-1D}

We further simulate a two-dimensional WCA liquid in a narrow channel, and obtain the identical scaling exponent for different one-dimensional packing fractions $\varphi_{1 \mathrm{D}}=\left(N / L_{c}\right) \sigma$ for $N$ particles in a channel of lateral length $L_{c}>N \sigma$ and accessible transverse slit width $L \ll \sigma$. The calculation for the relaxation rate can be adapted without conceptual changes also for narrow two-dimensional channels and yields

$$
\tau_{1 D}^{-1}=\frac{4}{3 \sqrt{\pi}}\left(\frac{L}{\sigma}\right)^{2} t_{0}^{-1} \frac{\varphi_{1 \mathrm{D}}}{1-\varphi_{1 \mathrm{D}}} .
$$

Here we used the fact that the contact value for a one-dimensional gas of hard particles is known exactly $g(\sigma)=$ $1 /\left(1-\varphi_{1 \mathrm{D}}\right)$ from the Tonks gas [59]. The simulation results are compared to the theoretical prediction for the divergence of the coupling time scale in the inset in Fig. 7. The prefactor calculated from the theory is shown also in Figure 7 and corroborates nicely the theoretical expectation. 


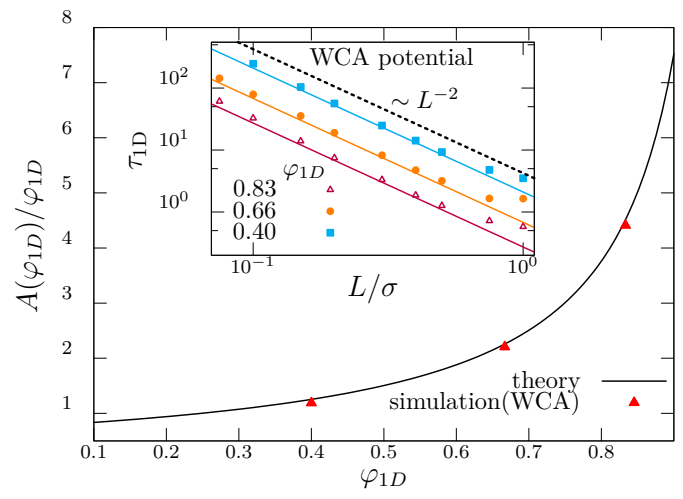

FIG. 7. Prefactor $A\left(\varphi_{1 \mathrm{D}}\right)$ for the WCA liquid extracted from the scaling behavior of the relaxation time $\tau_{1 \mathrm{D}}^{-1}\left(L, \varphi_{1 D}\right)=$ $t_{0}^{-1}(L / \sigma)^{2} A\left(\varphi_{1 D}\right)$ as a function of $1 D$ packing fraction $\varphi_{1 D}$. The inset shows the relaxation time $\tau_{1 \mathrm{D}}$ for the strongly confined WCA liquids as a function of the wall separation $L$ for various packing fractions $\varphi_{1 D}$. Packing fraction increases from top to bottom. The dashed line is a power law $\sim L^{-2}$ and serves as a guide to the eye. 